Indian SeCtion.

\title{
The Duties of a Nursing Superintendent in a Leprosy Settlement.
}

\author{
M. P. ThORNTON.
}

$\mathrm{O}$ $\mathrm{NE}$ of the chief duties of a sister in a leprosy settlement is to train others to nurse the sick as well as give the special treatment for leprosy. Sufferers from leprosy may have many other ailments in addition, and only under trained supervision can these be treated. For instance, in the Leprosy Homes at Purulia quite a number of the patients have severe anæmia, the result of hookworm in their systems. With the co-operation of the nurses and a dispenser for the microscopical examinations, during 1930, 150 of the worst cases were successfully treated, another 200 partially so. During the following year the remainder of the patients were examined and treated where necessary. No light task as there are over 800 people altogether.

A few enquiries made, brought to light the fact that many of the cases of leprosy had made a marked improvement. In March, the four homes for children suffering from leprosy were entirely cleared of hookworm trouble. The following December the Medical Superintendent remarked at the quarterly examination how surprisingly all the children had improved that year. One other striking case came under notice. Madan had received treatment for about five years. Every microscopic examination showed that he was still a positive case, the number of bacilli in the skin and mucosa lessening for a time, then remaining stationary for some period. In April, 1930, he was treated for hookworm, and after two months' treatment this was eliminated. Further tests for leprosy were found negative, and in December he was discharged from the Homes as "symptom free."

In every settlement there are many types of people; from these we can obtain our nurses for training. Personally, I am not in favour of using the healthy children in our Homes to train as nurses in the leprosy hospital. Patients of the cutaneous type of leprosy requiring hospital treatment are usually of the most infective type. Because of this, only nurses already trained to deal with contagious diseases are suitable, unless they already have the same disease. In Purulia, we had only ignorant village women who were willing to come forward for training for the Women's 
Hospital. In the Men's Hospital, matters were a little better, as these nurses could read and write in the vernacular.

Patience and perseverence overcame the illiteracy in the Women's Hospital. Two learnt to read thermometers and mark charts correctly. After a while, more intelligent girls were obtainable. Classes are held from time to time, of a simple nature, e.g., a talk on anatomy dealing perhaps with some special point occurring in some particular disease prevalent at the time, e.g., cases of pneumonia provide a good opportunity to give a lecture on the lungs that may be remembered. The illiterate nurse has her interest aroused, and is, therefore, enabled to nurse her patient more intelligently. She will also probably retain more than a little of the knowledge thus obtained. With the more literate, notebooks can be used. Talks on hygiene, medicines, diets are appreciated, if not given too frequently. This, of course, does not apply to the more educated nurse, who is able to attend frequent regular classes. Theatre duties are taught in the operating theatre after the essentials of surgical asepsis have been explained. In spite of the latter, I found the nurses had not really grasped surgical asepsis until they had actually assisted in the theatre. To have all new nurses in the theatre for a start was a trifle difficult, but by giving each one a certain duty and making them understand they had no other duty to think of, they managed very well. At present, six out of our staff of eighteen can now take any part of theatre duties, four being able to assist the surgeon, if necessary.

Practical classes for bandaging are held, to learn the correct method of special bandaging. I found the nurses very apt with simple methods for hands and feet, even if not the approved method, which would not always answer on some of the queer shaped stumps we get in the advanced nerve cases.

As previously mentioned, studies of special diseases lead to more intelligent nursing. Malarial patients have their quinine on the strike of time, remaining under nets from 5 p.m. until 5 a.m., hookworm cases are followed up with iron tonics, repeated treatments and examinations where necessary, fourteen doses being our highest number for one case. Nurses are responsible for looking up any defaulters. In the case of tuberculosis they have learnt the danger of infection, and report any person in the Homes known to have a troublesome morning cough. These can then be examined and isolated in the Tubercular Hospital if necessary. Where this has been done the house of the patient is then whitewashed, and a nurse is responsible for the 
thorough disinfection of the floor before the other inmates return to it. I have always found these duties carried out, the nurses responding well to the responsibility given to them. In dealing with the septic troubles, again the trained nurses' teaching is invaluable.

Studying the necessity for cleanliness in small details prepares the way for the asepsis necessary when giving the injection treatment for leprosy. A nurse who has had a brief time in hospital training will prove more apt when sent to work in the treatment department. Owing to the chronic nature of the nursing chiefly required in hospital for leprous patients it is possible to let the nurses work also in the treatment department. For instance, women injectors are better for the treatment of women and it is possible for the more educated female nurses to attend to this work, the less intelligent remaining in hospital to attend to the duties, these carrying out the day's routine. The nurses know, and have seen the results from an injection badly given, and may have had to attend to the sufferer day after day, and this tends to make them more careful. Also their lectures on the necessity for cleanliness and asepsis make them appreciate the technique of their work more than if merely learning " how" to give an injection.

Another side of the trained nurses work is Child Welfare. Mothers admitted with infants are immediately looked up, the children then moved to separate homes where these are provided. Mothers are usually quite ready to give up the little ones within three days of their arrival when they see and hear a little of the care that will be given to their children.

Another duty falling to the superintendence of a trained nurse is the regular treatment of the eyes, ears, nose and throat cases. Before the former clinic was commenced in Purulia by the late Dr. Landeman and Sister Grimshaw, eye cases had been treated sporadically in either hospital or dispensary, attendance being very irregular, as patients did not like waiting their turn, neither would they all come early to have treatment before the ordinary dressings began. Now they have their special clinic, opened for a certain time each morning. Later in the day the same room is used for the other special clinics. Working in these places we have a number of workers who are themselves sufferers from leprosy and are able to do the necessary treatment under a Sister's supervision.

The teaching of games and exercises for the girls should not be excluded. Boys are naturally energetic, act on their own initiative and obtain plenty of exercise by means of 


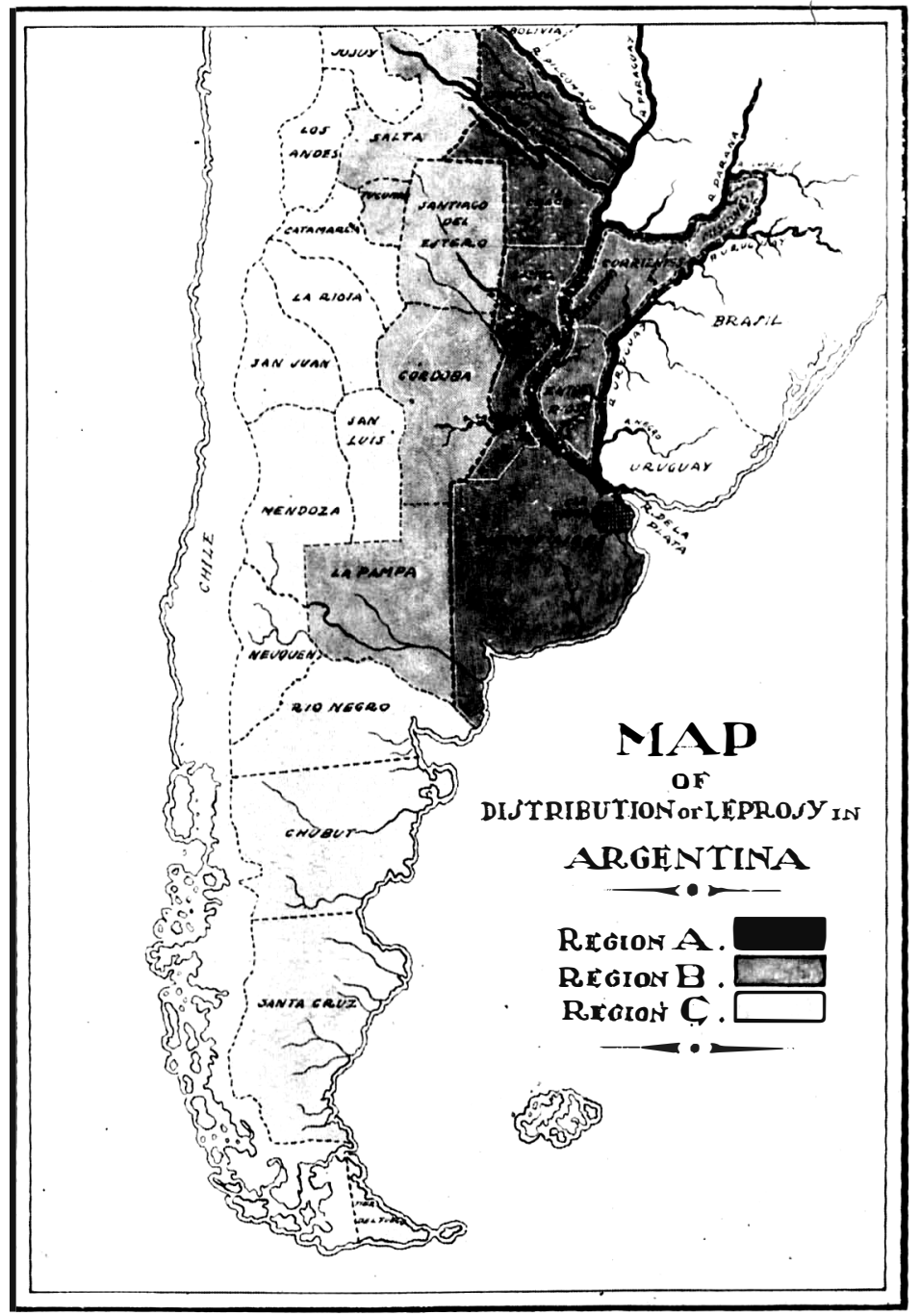




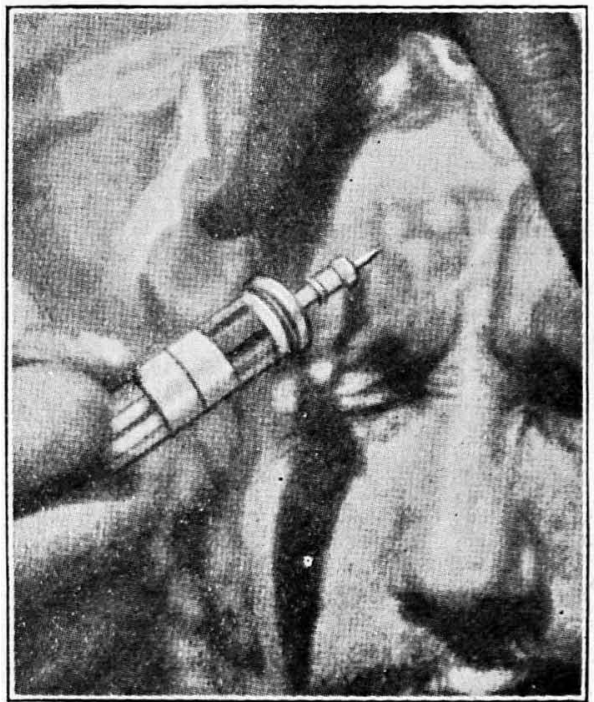

No. 1.-Inflltration OF SKIN OF Forehead; NOte Wheals.

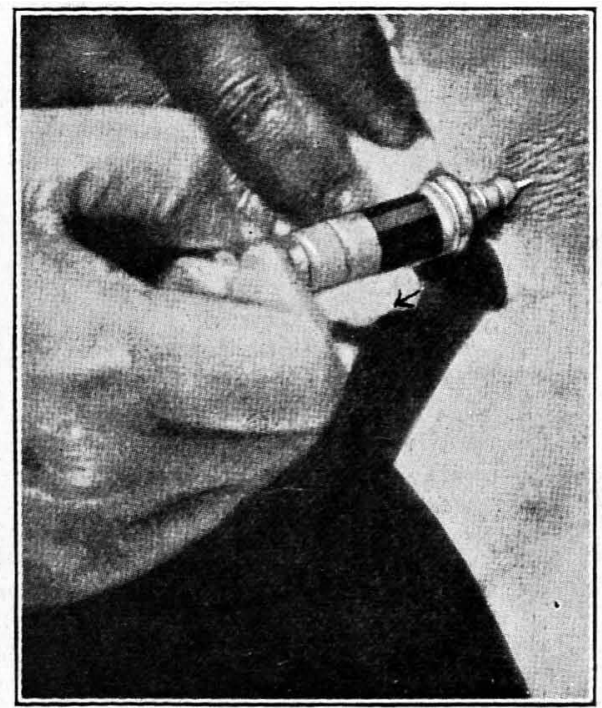

No. 3.-Infiltration of Thickened SKIN ; NOTE SKIN Markings Standing Out.

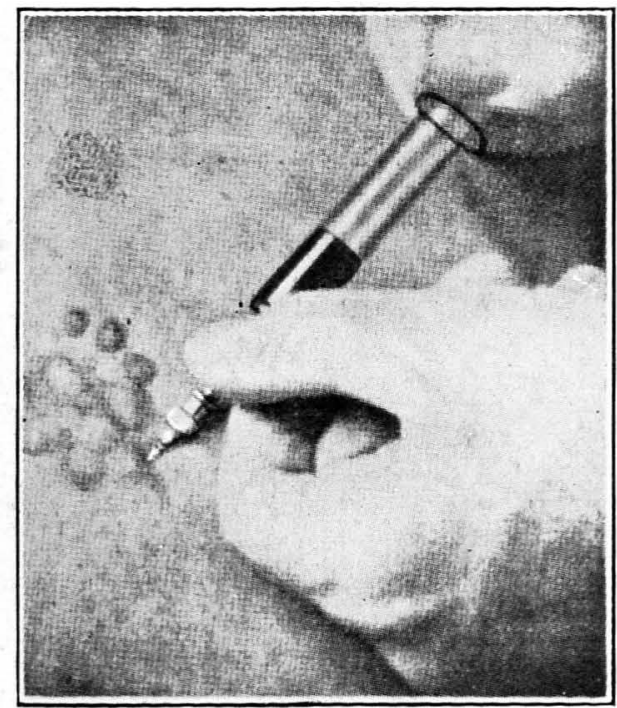

No. 4.-Infiltration of Skin of BACK ; NOTE Wheals.

By kind permission of Dr. E. Muir. 
gardening, games, \&c. Girls are more inclined to sit about and need encouraging to exercise. Games need to be taught, and other interest aroused. In this cause great benefit can be obtained through the formation of a Girl Guide company, the activities graded according to the ability of the girls. This branch of the work could certainly be undertaken by a general or educational worker, but in a home where only trained nurses are on the staff of women workers it is a detail not to be overlooked. 